\title{
O EFEITO DA SUGESTÃO DE FALSA INFORMAÇÃO PARA EVENTOS EMOCIONAIS: QUÃO SUSCETÍVEIS SÃO NOSSAS MEMÓRIAS? ${ }^{1}$
}

\author{
Carmem Beatriz Neufeld \\ Priscila Goergen Brust ${ }^{\#}$ \\ Lilian Milnitsky Stein
}

\begin{abstract}
RESUMO. O presente trabalho visa fornecer evidências científicas da susceptibilidade das memórias a distorção através do estudo das falsas memórias (lembranças de eventos que, na realidade, não ocorreram) para situações emocionalmente carregadas. Foi usada a versão brasileira dos instrumentos e procedimentos de Cahill, Prins, Weber, e McGaugh e adaptada por Neufeld, Brust e Stein para a investigação do efeito da emoção na memória e nas falsas memórias. O experimento investigou o efeito de sugestão de falsa informação em 138 alunos universitários. O procedimento também testou um material de sugestão de falsa informação congruente com a história na forma de um artigo de jornal. Os resultados indicam que a memória verdadeira não foi imune a erros e distorções, apesar de aparentemente ter sido auxiliada pela emocionalidade do evento. Ou seja, a sugestão de falsa informação parece ter auxiliado na discriminação dos itens sugeridos.
\end{abstract}

Palavras-chave: falsas memórias, emoção, sugestionabilidade.

\section{MISINFORMATION EFFECT ON MEMORY FOR EMOTIONAL EVENTS: HOW SUSCEPTIBLE ARE OUR MEMORIES?}

\begin{abstract}
The current essay forwards scientific evidence on the susceptibility of distorted memories by assessing false memories (remembrance of events which actually did not occur) in emotionally arousing situations. The Brazilian version of the Cahill, Prins, Weber and McGaugh's instruments and procedures adapted by Neufeld, Brust and Stein on the evaluation of the effect of emotion on memory and false memories were used. Misinformation effect on memory of 138 college students has been investigated. Misinformation material congruent to the story given in a newspaper article has also been tested. Results suggest that true memory has not been immune from errors and distortions even though it has apparently been supported by emotionally arousing events. In fact, misinformation seems to have assisted memory in the discrimination of the items suggested.
\end{abstract}

Key words: False memories, emotion, suggestibility.

\section{EL EFECTO DE INFORMACIÓN ENGAÑOSA PARA EVENTOS EMOCIONALES: ¿CUÁN SUSCEPTIBLES SON NUESTRAS MEMORIAS?}

\footnotetext{
RESUMEN. El presente trabajo tiene el objetivo de proporcionar evidencias científicas de la susceptibilidad de la memoria y su distorsión a través del estudio de las falsas memorias (recuerdo de eventos que en realidad, no ocurrieron) para situaciones emocionalmente intensas. En este estudio se utilizó la versión brasileña de los instrumentos y procedimientos Cahill, Prins, Weber y McGaugh adaptada por Neufeld, Brust y Stein para la investigación del efecto de la emoción en la memoria y las falsas memorias. El experimento investigo el efecto de sugestión de falsa información en 138 alumnos universitarios. El procedimiento también evaluó un material de sugestión de falsa información congruente con la historia en forma de un artículo de periódico. Los resultados indican que la memoria verdadera no fue inmune a errores y distorsiones a pesar de aparentemente haber sido auxiliada para la emocionalidad del evento, o sea, la sugestión de la falsa información parece haber auxiliado la discriminación de los ítems sugeridos.

Palabras-clave: Falsas memorias, emoción, sugestibilidad.

Apoio: CAPES e FAG.

* Psicóloga. Doutora em Psicologia. Docente da Universidade de São Paulo.

* Psicóloga. Mestranda em Cognição Humana, Pontifícia Universidade Católica do Rio Grande do Sul.

If Psicóloga. Doutora em Cognitive Psychology. Professora da Pontifícia Universidade Católica do Rio Grande do Sul.
} 
Diversas pesquisas na literatura referente à memória em adultos têm se devotado ao estudo da falsificação da memória, principalmente em casos pertinentes a questões clínicas (Mazzoni, Loftus \& Kirsch, 2001; Pergher, Stein \& Wainer, 2004) e jurídicas (Eisen, Morgan \& Mickes, 2002; Loftus, 2003; Nygaard, Feix \& Stein, 2006; Stein \& Memon, 2006). Assim, na última década, os avanços das pesquisas em Psicologia Experimental Cognitiva têm evidenciando que o transcurso do tempo pode transformar as lembranças (Stein \& Nygaard, 2003). As falsas memórias são lembranças vívidas de eventos que, na realidade, não ocorreram (Brainerd \& Reyna, 2005).

Uma das teorias que tem se lançado em busca da explicação do fenômeno das falsas memórias na atualidade é a Teoria do Traço Difuso (Reyna \& Brainerd, 1995), para a qual a memória não é um sistema unitário - pelo contrário, existem dois sistemas independentes de memória, processados paralelamente: a memória literal e a de essência. A memória de essência é ampla, robusta e armazena somente as informações inespecíficas, ou seja, aquelas que representam o significado da experiência como um todo. Já a memória literal é a codificação das informações de forma precisa, registrando e armazenando detalhes, sendo, contudo, mais suscetível ao esquecimento e à interferência, se comparada à memória de essência (para uma revisão, ver Brainerd \& Reyna, 2005; Stein \& Neufeld, 2001).

Além disso, as falsas memórias podem ocorrer de duas formas: espontaneamente ou via implantação de sugestão de falsa informação (Brainerd \& Reyna, 2002). As falsas memórias que são geradas espontaneamente resultam do processo normal de compreensão, ou seja, são fruto de processos de distorção mnemônica endógena (Stein \& Neufeld, 2001). Já o procedimento de sugestão de falsa informação consiste na apresentação de uma informação falsa compatível com a experiência, que passa a ser incorporada na memória sobre esta vivência (Lindsay, Hagen, Read, Wade \& Garry, 2004). Esta distinção é fundamental, especialmente em situações em que se espera que a pessoa relate suas experiências de forma fidedigna, ao invés de suas inferências ou entendimentos sobre o que ocorreu, como no caso de um testemunho num tribunal (Brainerd, Reyna \& Poole, 2000; Ceci \& Bruck, 1993).

No entanto, desde o início da década de 90 diversos pesquisadores apresentaram críticas contundentes aos estudos de falsas memórias em geral e às pesquisas sobre efeito da sugestão de falsa informação em específico (Hudson, Fivush \& Kuebli, 1992; Raskin \& Esplin, 1991; Saywitz, Goodman, Nicholas \& Moan, 1991). Tais críticas giram em torno, fundamentalmente, das características das tarefas utilizadas como material-alvo nos estudos de falsas memórias até então. Howe (1991), por exemplo, considera que os materiais a serem recordados não seriam representativos das informações relevantes com as quais nos deparamos no nosso dia-a-dia, portanto as falsas memórias, tanto as espontâneas quanto as sugeridas, ocorreriam para fatos periféricos da vida das pessoas.

Neste sentido, tarefas excessivamente fáceis não utilizariam recursos atencionais suficientes para serem memoráveis, gerando assim um decréscimo na acurácia da memória para eventos cotidianos e menos importantes (Howe, 1991; Hudson et al., 1992). Os autores argumentam, igualmente, que a suscetibilidade à sugestão de falsa informação não ocorreria para a memória de eventos realmente vivenciados pelo sujeito, aos quais sentimentos estariam relacionados. Sendo assim, as memórias relacionadas a situações traumáticas ou emocionalmente carregadas estariam imunes a tais erros de memória.

Dados recentes da literatura discordam dessa imunidade da memória para situações emocionais, evidenciando efeito de sugestão tanto para situações cotidianas (Braun, Ellis \& Loftus, 2002; Lindsay, Allen, Chan \& Dahl, 2004) como para aquelas relativas a eventos autobiográficos (Safer, Levine \& Drapalski, 2002). Assim, desconsidera-se a hipótese de que a memória para eventos emocionalmente intensos (i.e., ideação suicida, depressão grave, abuso sexual, guerra) não pode ser distorcida. Não obstante, tal efeito encontra-se pouco explorado na literatura (Klimes-Dougan, 1998; Lindsay \& Read, 1994; Neufeld, Brust \& Stein, no prelo). Destarte, permanece em aberto a questão a ser testada referente à suscetibilidade à sugestão para um evento emocionalmente carregado em condições experimentais controladas.

Devido às fortes implicações clínicas dos estudos de emoção e falsas memórias, observa-se um aumento crescente de trabalhos que focam sua amostra em grupos clínicos (Pergher, Grassi-Oliveira, Ávila \& Stein, 2005; Pergher, Grassi-Oliveira \& Stein, 2005). Nesse sentido, em um estudo com pacientes deprimidos (Ellis, Ottaway, Varner, Becker \& Moore, 1997), estes se mostraram mais suscetíveis a falsas memórias do que pacientes não-deprimidos. Em contrapartida, tais participantes também obtiveram uma melhor performance nas lembranças verdadeiras. Isto significaria dizer que os participantes deprimidos 
tiveram uma performance superior à dos participantes do grupo-controle em memórias verdadeiras, associada, no entanto, a um índice maior de falsas memórias.

O contrário parece ocorrer com os participantes expostos a altos níveis de ansiedade. Barbosa (2002) testou a memória semântica de estudantes universitários minutos antes de um exame bimestral. Na comparação dos escores da Escala de Ansiedade Traço e Estado (Idate) com a performance no teste de memória, os dados revelaram uma perda significativa na memória, diretamente proporcional aos escores alcançados pelos participantes na Idate. Estes dados sugerem que, sob fortes níveis de ansiedade, a memória torna-se mais suscetível a distorções (Barbosa, 2002).

Tomando por base a literatura revisada em relação à falsificação da memória e à emoção, o presente trabalho buscou investigar o efeito da sugestão de falsa informação sobre a memória para evento emocional. Para tanto, aplicou-se um instrumento que possibilitasse o acesso a memórias de evento emocional, utilizando-se a versão brasileira adaptada por Neufeld et al. (no prelo), do procedimento de Cahill, Prins, Weber e McGaugh (1994), aprimorado por Cahill e McGaugh (1995) e traduzido para o português por Quevedo et al. (2003).

\section{MÉTODO}

\section{Delineamento experimental}

$\mathrm{O}$ presente estudo envolveu um delineamento experimental fatorial misto $2 \times 2 \times 3 \times 3$ com medidas repetidas nas duas últimas variáveis. A primeira variável, emocionalidade do material-alvo (emocional e não-emocional), e a segunda variável, sugestão de falsa informação (com e sem), foram manipuladas entre grupos. As demais variáveis foram as três fases do material-alvo e os três tipos de itens do teste de memória (alvo, relacionado e não-relacionado).

A partir disto, foram criados quatro grupos de participantes, que receberam diferentes tratamentos. Os Grupos 1 e 2 compunham o grupo experimental, cujo material-alvo versava sobre uma situação considerada emocionalmente carregada. Os dois diferiam entre si apenas pelo fato de que o Grupo 1 recebeu sugestão de falsa informação, enquanto o 2 não foi exposto à sugestão. $\mathrm{O}$ mesmo procedimento foi realizado em relação aos Grupos 3 e 4, visto que, enquanto o Grupo 3 foi exposto à sugestão de falsa informação, o Grupo 4, não. Estes dois últimos grupos foram denominados de grupo-controle, visto que o material-alvo deste grupo relatava uma situação considerada não carregada emocionalmente.

As variáveis dependentes foram a performance da memória (verdadeira, falsa e de viés) e a avaliação subjetiva do nível da emoção despertada pelo materialalvo (emocional, neutra e não-emocional). Foram realizados levantamentos dos escores de ansiedade e depressão, no intuito de controlar a ação destas variáveis sobre os diferentes grupos experimentais.

\section{PARTICIPANTES}

A amostra inicial foi composta por 160 estudantes universitários pertencentes a diversos cursos de graduação (ciências biológicas, administração, farmácia, enfermagem, nutrição) de uma instituição privada de ensino superior do Paraná. Como o presente estudo integra duas sessões experimentais ocorreu uma perda amostral de 22 participantes que não compareceram à segunda sessão de testagem. A amostra definitiva ficou então composta de 138 estudantes, sendo 51 do sexo masculino e 87 do sexo feminino, com idades entre 17 e 44 anos $(M=23,51$, $D P=6,67)$. A formação dos grupos deu-se através de designação aleatória, de forma que a cada encontro dos participantes, uma das versões da história foi apresentada. $\mathrm{O}$ grupo experimental com sugestão foi composto de 32 participantes (15 homens), o grupo experimental sem sugestão foi composto de 35 participantes ( 7 homens), enquanto 37 estudantes (14 homens) compuseram o grupo-controle com sugestão e 34 estudantes (15 homens) compuseram o grupocontrole sem sugestão.

\section{Instrumentos}

O material-alvo (Neufeld et al., no prelo) foi adaptado do material original de Cahill et al. (1994) traduzido por Quevedo et al. (2003), e era composto de uma seqüência de 11 slides divididos em três fases. A fase 1 (slides 1 a 4) apresentava uma mãe e seu filho a caminho do hospital e a foto do pai em um laboratório; a fase 2 (slides 5 a 8) apresentava um carro batido e diversos procedimentos hospitalares; já a fase 3 (slides 9 a 11) apresentava a mãe caminhando na rua, ao telefone $\mathrm{e}$ indo embora. $\mathrm{O}$ grupo experimental e o grupo-controle receberam a mesma seqüência de slides.

Todavia, foram apresentadas duas versões narrativas da história. A narrativa referente aos slides das fases 1 e 3 também foi a mesma para os dois grupos. $\mathrm{O}$ que diferenciou um grupo do outro foi que, no grupo experimental, as frases referentes aos slides 
da fase 2 relatavam um acidente que causava graves ferimentos no menino, enquanto na mesma fase, no grupo-controle, foi relatado que o menino viu um carro batido e assistiu a um treinamento de atendimento de urgência no hospital onde o pai trabalhava. As duas versões da história foram gravadas em CD, narradas por uma estudante de comunicação social em fase de conclusão de curso, com o cuidado de manter fixo o intervalo de 6 segundos para a apresentação de cada slide.

A escala subjetiva de emoção consistia de duas questões. A primeira foi: "A história que você acabou de ouvir causou alguma emoção em você?", com opções "sim" e "não", conforme a escolha do participante. A segunda questão solicitava: "Em caso afirmativo, marque um $\mathrm{X}$ no número correspondente ao nível de intensidade da emoção que você sentiu ao ouvir a história e ver os slides apresentados anteriormente.". Logo abaixo foi apresentada uma escala Likert de 5 pontos, variando de "quase nenhuma" (1) até "extrema" (5).

O material de sugestão, desenvolvido para este estudo, consistia de dois textos que foram entregues aos participantes como se fosse uma notícia de jornal. O texto foi desenvolvido com base em uma notícia sobre acidentes de trânsito (BBCBrasil, 2004) contendo estimativas sobre acidentes e mortes no trânsito. A única diferença entre as duas notícias foi que o material de sugestão continha duas frases relacionadas ao acidente relatado no material-alvo experimental (e.g., no material-alvo era apresentado um CARRO AZUL, o material de sugestão apresentava a informação de que o carro era VERMELHO). Metade dos participantes recebeu a notícia com sugestão e a outra metade recebeu a notícia sem a sugestão de falsa informação.

O teste de memória de reconhecimento era autoadministrado e composto por 25 itens. Os itens do teste pertenciam a três categorias: alvo, relacionado e não-relacionado. A medida referente à condição de memória verdadeira era representada pelos itens-alvo, enquanto às falsas memórias correspondiam os itens relacionados, e os itens não-relacionados, à medida de viés (ou "chute"). Além disto, esses itens foram distribuídos proporcionalmente em relação às três fases do material original. Assim sendo, dos 10 itensalvo, 3 foram referentes à fase 1,4 à fase 2, e 3 à fase 3. Já os itens relacionados foram distribuídos entre as 3 fases, 2 para cada, perfazendo um total de 6 itens. Os itens não-relacionados representavam 9 do total. A distribuição dos itens nos testes de memória foi pseudo-aleatória, uma vez que os itens referentes à fase 2 mantiveram a mesma numeração para ambos os grupos, considerando a exposição ao evento emocionalmente carregado ou não.

As instruções do teste contavam com explicações detalhadas indicando aos participantes que marcassem um "X" na opção que correspondia exatamente àquilo que tinham visto nos slides ou ouvido na narrativa da história. Especificamente foi dito aos participantes: "Baseie suas respostas apenas na sua memória dos slides que viu e do relato da história que ouviu". Em seguida, destacava-se que deveria ser assinalado "não" para todas as opções que diferiam do que havia sido visto ou ouvido, mesmo que as alternativas oferecessem opções que pudessem ser inferidas a respeito do evento. Este cuidado era necessário, uma vez que os acertos representavam o índice de memória verdadeira, e as inferências, o de falsas memórias. Foi apresentado um item instruindo o participante sobre como responder o teste. Além disso, foi também solicitado ao participante que respondesse as questões na ordem em que elas aparecessem no teste, não deixando nenhuma questão em branco, nem retornando às questões anteriores.

Além disto, por se tratar de um material-alvo emocionalmente carregado, foram utilizados como variáveis de controle os índices de ansiedade e depressão testados através do Inventário de Ansiedade Beck - BAI (Beck \& Steer, 1993a) em sua versão brasileira (Cunha, 2001) e do Inventário de Depressão Beck - BDI (Beck \& Steer, 1993b) em sua versão brasileira (Cunha, 2001). Os inventários são autoadministrados, com duração média de aplicação de 20 minutos, e se referem a exemplos de comportamentos comuns ocorridos na última semana. O BAI consiste de 21 sintomas e é respondido pelo participante de acordo com uma escala Likert de 4 pontos (absolutamente não, levemente, moderadamente, gravemente). $\mathrm{O}$ BDI consiste de 21 grupos de afirmações. Cada grupo é numerado de 0 a 3 . O participante pode escolher uma das quatro afirmações pertinentes a cada um dos 21 grupos.

\section{Procedimentos}

A coleta de dados foi realizada em duas sessões experimentais, sendo que os participantes foram testados em grupo, em sala silenciosa, com a utilização de equipamento de multimídia para projeção dos slides e de aparelho de som para reprodução do $\mathrm{CD}$ com as versões experimental e controle, das histórias. O trabalho era apresentado aos participantes em suas próprias salas de aula, em períodos de aula cedidos pelo docente responsável pela disciplina, como uma pesquisa sobre a opinião dos estudantes quanto ao material que lhes seria apresentado, 
indicando que haveria um segundo encontro com o mesmo objetivo.

Os procedimentos relativos à ética na pesquisa foram tomados em conformidade com a legislação nacional, tendo sido aprovados pelo Comitê de Ética em Pesquisa da PUCRS. Sendo assim, após essa breve explicação, foi assegurada ao participante a liberdade para desistir a qualquer momento da pesquisa, e somente aqueles que assinaram o Termo de Consentimento Livre e Esclarecido participaram da pesquisa. Foi então solicitado aos participantes que prestassem bastante atenção na seqüência de slides e na história que iriam assistir, frisando-se a importância de eles evitarem fazer comentários ao longo dos procedimentos. Logo após a apresentação do materialalvo, foi entregue aos participantes a escala subjetiva de emoção, tendo-lhes sido solicitado que respondessem com o máximo de sinceridade sobre a intensidade que atribuíam à emoção despertada pelo material-alvo. Ao final da primeira sessão, os participantes responderam aos Inventários Beck de Ansiedade e Depressão, seguindo as instruções de marcarem as respostas de como se sentiram ao longo da última semana.

$\mathrm{Na}$ segunda sessão experimental, duas semanas após a apresentação do material-alvo, foi entregue aos participantes o material de sugestão. Foi dito aos participantes do grupo controle que o material referiase a uma notícia de jornal sobre acidentes de trânsito. Já para o grupo experimental, a notícia era apresentada como uma informação adicional sobre a história que eles tinham ouvido e visto na primeira sessão. Imediatamente em seguida, foi esclarecido que a memória para aquele evento seria testada, e os participantes foram submetidos ao teste de memória de reconhecimento auto-administrado. Foi salientada a importância de responder ao teste de memória com base nos slides e na história apresentada anteriormente. Além disto, foi solicitado aos participantes que lessem as instruções com atenção e permanecessem em silêncio após o término do teste, até que todos do grupo o tivessem concluído.

\section{RESULTADOS E DISCUSSÃO}

Todas as informações coletadas foram armazenadas em um banco de dados elaborado especialmente para este fim e analisadas com o auxílio do programa Statistical Package for Social Sciences (SPSS), versão 12.0 (Nie, Hull \& Bent, 2003). Todos os tratamentos estatísticos utilizaram um $\alpha<0,05$ para o teste de hipóteses. Foram realizadas análises post hoc e comparações pareadas com correção de Bonferroni. Não foram observadas diferenças entre grupos para o sexo dos participantes, assim todos os grupos foram considerados equivalentes.

\section{Indicadores emocionais}

Com o intuito de controlar a influência das variáveis ansiedade e depressão sobre os diferentes grupos de pesquisa, os escores apresentados pelos participantes nos Inventários de ansiedade e depressão (BAI e BDI) foram testados através de uma ANOVA Multivariada para grupo (experimental vs. controle). Não foram encontradas diferenças significativas entre grupo experimental e grupo-controle nos índices de ansiedade e depressão ( $p s>0,05)$, indicando uma distribuição homogênea destas variáveis nos grupos pesquisados. Os escores médios no BAI no grupo controle $(M=13,75 ; D P=10,976)$ foram semelhantes aos do grupo experimental $(M=12,79 ; D P=7,751)$. No que se refere à depressão, os índices mantiveramse constantes entre os grupos controle $(M=10,71 ; D P$ $=8,871)$ e experimental $(M=9,88 ; D P=6,338)$.

Os resultados da escala subjetiva de emoção (escala Likert de 5 pontos) foram testados através de uma ANOVA Univariada para grupo (experimental vs. controle) $\mathrm{x}$ sugestão (com sugestão vs. sem sugestão). A análise evidenciou efeito principal de grupo $(F(1,137)=17,965, p<0,001)$. Os participantes do grupo experimental $(M=2,41, \quad D P=1,38)$ avaliaram o material-alvo com maior nível emocional do que os participantes do grupo-controle $(M=1,43$, $D P=1,33)$. Estes resultados sugerem que $o$ experimento obteve sucesso na manipulação da variável emoção.

\section{Indicadores de memória}

A avaliação da performance da memória dos participantes foi baseada em três tipos de resposta: (1) $\mathrm{MV}$ : foram consideradas memórias verdadeiras todos os reconhecimentos, ou seja, as respostas "sim" para os itens-alvo do teste de memória; (2) FM: foram consideradas falsas memórias todas as respostas "sim" para os itens relacionados do teste de memória; (3) $\mathrm{RV}$ : consideraram-se como respostas de viés todas as respostas "sim" para os itens não-relacionados no teste de memória.

Considerando-se os resultados de memória geral, as respostas para o teste de memória foram analisadas através de uma ANOVA para grupo (experimental vs. controle) x sugestão (com sugestão vs. sem sugestão) $\mathrm{x}$ item (alvo vs. itens relacionados vs. itens nãorelacionados), com medidas repetidas na última variável. Esta ANOVA evidenciou efeito principal 
apenas para a variável item $(F(2,136)=253,847, p<$ $0,001)$. Foi encontrada uma diferença marginalmente significativa $(p=0,053)$ entre o índice de $\operatorname{MV}(M=$ $0,591, D P=0,188)$ e o índice de FM $(M=0,533, D P$ $=0,205)$, sendo o primeiro superior ao segundo. Já o índice de RV $(M=0,137, D P=0,176)$ foi significativamente inferior ( $p s<0,01)$ tanto aos índices de MV quanto aos de FM.

Os dados apontados pela literatura (Brainerd \& Reyna, 1995, 2002) indicam que, em testes de reconhecimento, usualmente, alvos são mais aceitos do que itens relacionados, e itens relacionados são mais aceitos do que itens não-relacionados. No entanto, observando-se os resultados, percebe-se que os índices de MV e FM são semelhantes, o que pode ser atribuído ao fato de o teste de memória ter sido realizado duas semanas após a apresentação do material-alvo. Estudos utilizando o mesmo procedimento (Quevedo et al., 2003) indicam que a memória verdadeira para eventos emocionalmente carregados apresenta índices maiores de reconhecimento em um momento posterior à exposição ao evento do que em um momento imediatamente após. Pressupõe-se um efeito semelhante com relação às falsas memórias, uma vez que a memória de essência é mais duradoura que a literal (Stein \& Neufeld, 2001).

Diversos estudos indicam que os índices de FM aumentam proporcionalmente ao intervalo de tempo entre a apresentação do material-alvo e o teste de memória (Neufeld \& Stein, 2003; Reyna \& Brainerd, 1995); porém os estudos citados foram realizados sem a manipulação da variável emoção, ou seja, os resultados de que os índices de FM se assemelharam aos índices de MV em um teste de memória posterior são condizentes com a literatura de falsas memórias em geral.

O efeito principal da ANOVA foi qualificado por uma interação marginalmente significativa entre item e sugestão $(F(2,136)=2,919, p=0,057)$. Considerandose os índices de MV e de RV, não foram observadas diferenças significativas entre o grupo que recebeu sugestão e o grupo que não recebeu sugestão $(F s(1,133)<1,207, p s>0,05)$. Em relação aos índices de $\mathrm{FM}(F(1,133)=4,109, p>0,05)$, ao contrário do esperado, os índices do grupo com sugestão $(M=$ $0,500, D P=0,201)$ foram inferiores aos do grupo sem sugestão $(M=0,572, D P=0,200)$.

Encontram-se na literatura dados semelhantes aos citados acima, onde a sugestão parece ter auxiliado na discriminação dos itens sugeridos, diminuindo o índice de falsos reconhecimentos. Nesse sentido, alguns estudos (Ghetti \& Goodman, 2001; Stein \& Reyna, 1997) sugerem que pode haver situações em que a confiança para memórias verdadeiras e falsas aumenta quando da apresentação de sugestão, e assim aumenta também o reconhecimento de informações falsas e estas se distinguem das verdadeiras. No presente estudo, a sugestão parece ter deixado os participantes mais atentos aos itens no teste de memória, auxiliando no reconhecimento das informações falsas sugeridas.

Além disto, neste trabalho a sugestão foi apresentada na mesma sessão experimental em que a memória dos sujeitos foi testada. Outros estudos (Titcomb \& Reyna, 1995) apresentam evidências da influência do tempo na sugestibilidade, ou seja, observaram que quando a sugestão foi apresentada uma semana após o materialalvo ocorria um acréscimo nos índices de falsas memórias sugeridas, se comparados aos índices obtidos na semana anterior. Frost (2000) testou o momento da sugestão apresentando a falsa informação 10 minutos e 1 semana antes do teste de memória. Os resultados evidenciaram um aumento das falsas memórias com o passar do tempo. Desta forma, o efeito da sugestão no presente estudo pode ter sido minimizado, devido ao fato de sua apresentação ter sido feita imediatamente antes do teste de memória.

As respostas para o teste de memória foram analisadas através de uma ANOVA para grupo (experimental vs. controle) $\mathrm{x}$ fase (fase $1 v s$. fase $2 v s$. fase 3) $\mathrm{x}$ item (alvo vs. itens relacionados vs. itens não-relacionados), com medidas repetidas nas duas últimas variáveis. Foram evidenciados dois efeitos principais, um para item $(F(2,133)=246,119, p<$ $0,001)$ e outro para fase $(F(2,133)=34,234, p<$ $0,001)$. A ANOVA também indicou interações entre fase e grupo $(F(2,133)=4,751, p<0,01)$, e entre item e fase $(F(2,133)=25,284, p<0,001)$. Tais efeitos foram qualificados pela interação entre item, fase e grupo $(F(2,133)=2,438, p<0,05)$. Na Tabela 1 são apresentados os resultados desta última interação.

Tabela 1. Médias de Reconhecimento por Tipo de Item para Cada uma das Fases por Grupo (Desvio Padrão entre Parênteses)

\begin{tabular}{|c|c|c|c|}
\hline & MV & FM & $\mathbf{R V}$ \\
\hline \multicolumn{4}{|l|}{ Fase 1} \\
\hline Experimental & $0,482(0,280)$ & $0,704(0,339)$ & $0,227(0,281)$ \\
\hline Controle & $0,565(0,272)$ & $0,647(0,352)$ & $0,183(0,230$ \\
\hline
\end{tabular}

Fase 2

Experimental $0,814(0,260) * 0,507(0,366) \quad 0,106(0,235)$

Controle $\quad 0,704(0,284) * 0,401(0,383) \quad 0,131(0,235)$

Fase 3

Experimental $0,414(0,280) \quad 0,454(0,336) \quad 0,055(0,161)$

Controle $\quad 0,439(0,284) \quad 0,500(0,358) \quad 0,122(0,247)$

Nota. $\mathrm{MV}=$ memórias verdadeiras; FM = falsas memórias; RV = respostas de viés.

$* \mathrm{p}<0,001$ 
Em termos do grupo experimental (versão emocionalmente carregada do evento), houve uma maior ocorrência de MV na fase $2(p<0,001)$, não sendo detectada diferença significativa nos índices de MV entre a fase 1 e a fase $3(p>0,05)$. Em relação às FMs, os índices alcançaram os níveis mais elevados na fase $1(p<0,01)$, decrescendo na fase 2 e mantendo-se estáveis na fase $3(p>0,05)$. O mesmo efeito foi encontrado no que tange às RVs: a fase 1 obteve o maior índice em relação à fase $2(p<0,05)$ e à fase 3 ( $p<0,001)$, sendo que os índices não variaram entre as duas últimas fases $(p>0,05)$.

Quando consideradas as médias do grupocontrole (versão não emocional do evento), os índices de MV obtiveram dados semelhantes aos relatados para o grupo experimental, onde a fase 2 apresentou os maiores índices ( $p s<0,01)$. No entanto, os índices da fase 1 de MV foram superiores aos índices da fase $3(p<0,01)$. Já no que se refere aos índices de FM, ocorreu um decréscimo dos índices da fase 1 para a fase $2(p<0,001)$, no entanto, não houve diferença entre as fases 2 e 3 ( $p>$ $0,05)$. As RVs mantiveram índices constantes ao longo das três fases $(p s>0,05)$.

Comparando-se os índices dos grupos experimental e controle de acordo com a fase, foram evidenciadas diferenças significativas apenas para MV na fase 2, sendo que o grupo experimental obteve índices superiores aos do grupo-controle $(F(1,133)=$ $5,281, p<0,05)$. No que se refere às FMs e RVs, não foram detectadas diferenças nos índices dos grupos experimental e controle para nenhuma das três fases $(F s(1,133)<3,310, p s>0,05)$.

Levando-se em consideração os resultados obtidos na comparação dos índices em cada um dos grupos, os dados sugerem um efeito potencializador da emoção sobre as MVs, já que a fase 2 pode ser considerada a parte mais emocionalmente carregada do evento. Estes resultados são congruentes com os encontrados na literatura para as MVs (Cahill \& McGaugh, 1995, 1996), onde os índices foram maiores para o grupo sob efeito de maior intensidade emocional em comparação ao grupo-controle. No entanto, a emoção não parece ter auxiliado na rejeição dos itens relacionados e dos itens não-relacionados. Isto sugere que a emoção não garante imunidade à memória contra distorções ou, até mesmo, contra a aceitação de itens sem relação alguma com o evento.

\section{CONSIDERAÇÕES FINAIS}

O presente estudo teve como objetivo principal verificar o efeito da sugestão de falsa informação sobre a memória de um evento emocionalmente carregado. Os resultados indicaram discrepância com boa parte dos estudos clássicos da literatura (Brainerd \& Reyna, 2002; Loftus, 1979). No presente estudo, não foi observado o clássico efeito da falsa informação, qual seja, diminuição dos índices de memórias verdadeiras e aumento das falsas memórias sugeridas; pelo contrário, os resultados parecem indicar que a apresentação da notícia com informações falsas pode ter deixado os participantes mais atentos aos itens sugeridos no teste de memória, ajudando-os a discriminar itensalvos de itens relacionados sugeridos. Resultados semelhantes foram encontrados por outros pesquisadores (Ghetti \& Goodman, 2001).

Além disto, a influência da variável momento de apresentação da sugestão deve ser considerada. $\mathrm{Na}$ vida cotidiana, a sugestão de falsa informação pode ser apresentada com diferentes intervalos temporais dias, meses ou até anos antes da recuperação do evento. No experimento em questão, no entanto, a sugestão foi apresentada imediatamente antes do teste de memória. Diversos estudos, como os de Frost (2000) e Warren e Lane (1995), por sua vez, apresentam evidências da potencialização do efeito da sugestão na suscetibilidade da memória, decorrente da influência do tempo entre a sugestão e o teste de memória. Sugere-se, portanto, que futuras investigações sejam realizadas testando diferentes momentos de apresentação da sugestão.

Outro foco do presente estudo refere-se aos dados da literatura que versam sobre a interface da emoção com a acurácia da memória. Diferentes autores (Howe, 1991; Raskin \& Esplin, 1991) e o próprio senso comum argumentam que lembranças de eventos emocionalmente carregados devem ser de natureza distinta das lembranças corriqueiras e cotidianas, sem carga emocional considerável, e que por isto mesmo, as memórias emocionais devem se conservar melhor com a passagem do tempo.

Os nossos resultados indicam que, apesar de as MVs aparentemente terem sido auxiliadas pela emocionalidade do evento, a memória não ficou imune a erros e distorções. Isto sugere que o simples fato de uma memória estar acompanhada de conteúdo emocional não é em si uma garantia de que ela se manterá fidedigna. Tal conclusão tem fortes implicações na Psicologia e em outras áreas da saúde, pois se contrapõe à idéia de que uma lembrança com forte carga emocional perdura intacta por anos. Além disto, informações sem relação alguma com o evento emocionalmente carregado podem ser recuperadas em índices superiores aos esperados. 


\section{REFERÊNCIAS}

Barbosa, C. (2002). Estudo experimental sobre emoção e falsas memórias. Dissertação de Mestrado não publicada, Pontifícia Universidade Católica do Rio Grande do Sul, Porto Alegre.

BBCBrasil (2004). Trânsito será terceira causa de morte ou ferimento até 2020, diz OMS, Online Journal of BBC Brasil: BBC Brasil. Recuperado em 22 de maio de 2004 de http://www.bbc.co.uk/portuguese/noticias/story/2004/04/040405_ omsebc.shtml

Beck, A. T. \& Steer, R. A. (1993a). Beck Anxiety Inventory Manual. San Antonio: Psychological Corporation.

Beck, A. T. \& Steer, R. A. (1993b). Beck Depression Inventory Manual. San Antonio: Psychological Corporation.

Brainerd, C. J. \& Reyna, V. F. (1995). Autosuggestibility in memory development. Cognitive Psychology, 28, 65-101.

Brainerd, C. J. \& Reyna, V. F. (2002). Fuzzy-trace theory and false memory. Current Directions in Psychological Science, 11(5), 164-169.

Brainerd, C. J. \& Reyna, V. F. (2005). The science of false memory. New York: Oxford.

Brainerd, C. J., Reyna, V. F. \& Poole, D. A. (2000). Fuzzy-trace theory and false memory: Memory theory in the courtroom. In D. F. Bjorklund (Ed.), False memory creation in children and adults: Theory, research, and implications (pp. 93-127). Hillsdale, N.J.: Erlbaum.

Braun, K. A., Ellis, R., \& Loftus, E. F. (2002). Make my memory: How advertising can change our memories of the past. Psychology \& Marketing, 19(1), 1-23.

Cahill, L. \& McGaugh, J. L. (1995). A novel demonstration of enhanced memory associated with emotional arousal. Consciousness and Cognition, 4, 410-421.

Cahill, L. \& McGaugh, J. L. (1996). Modulation of memory storage. Current Opinion in Neurobiology, 6, 237-242.

Cahill, L., Prins, B., Weber, M. \& McGaugh, J. L. (1994). ßAdrenergic activation and memory for emotional events. Nature, 371, 702-704.

Ceci, S. J. \& Bruck, M. (1993). The suggestibility of the child witness: A historical review and synthesis. Psychological Bulletin, 113, 403-439.

Cunha, J. A. (2001). Manual da versão em português das escalas Beck. São Paulo, SP: Casa do Psicólogo.

Eisen, L. E., Morgan, D. Y. \& Mickes, L. (2002). Individual differences in eyewitness memory and suggestibility: Examining relations between acquiescence, dissociation and resistance to misleading information. Personality and Individual Differences, 33, 553-571.

Ellis, H. C., Ottaway, S. A., Varner, L. J., Becker, A. S. \& Moore, B. A. (1997). Depressed mood, task organization, cognitive interference, and memory: Irrelevant thoughts predict recall performance. Journal of Social Behavior \& Personality, 12(2), 453-470.

Frost, P. (2000). The quality of false memory over time: Is memory for misinformation "remembered" or "known"? Psychonomic Bulletin \& Review, 7(3), 531-536.

Ghetti, S. \& Goodman, G. S. (2001). Resisting distortion. Forensic Psychology, 14(11), 58-59.
Howe, M. L. (1991). Misleading children's story recall: Forgetting and reminiscence of facts. Developmental Psychology, 27, 746762.

Hudson, J., Fivush, R. \& Kuebli, J. (1992). Scripts and episodes: The development of event memory. Applied Cognitive Psychology, 6, 483-505.

Klimes-Dougan, B. (1998). Screening for suicidal ideation in children and adolescents: Methodological considerations. Journal of Adolescence, 21(4), 435-444.

Lindsay, D. S., Allen, B. P., Chan, J. C. K., \& Dahl, L. C. (2004), Eyewitness suggestibility and source similarity: Intrusions of details from one event into memory reports of another event. Memory and Language, 50, 96-111.

Lindsay, D. S., Hagen, L., Read, J. D., Wade, K. A. \& Garry, M. (2004). True photographs and false memories. Psychological Science, 15(3), 149-154.

Lindsay, D. S. \& Read, J. D. (1994). Psychotherapy and memories of childhood sexual abuse: A cognitive perspective. Applied Cognitive Psychology, 8, 281-338.

Loftus, E. F. (1979). Eyewitness testimony. Cambridge, MA: Harvard.

Loftus, E. F. (2003). Our changeable memories: Legal and practical implications. Nature Reviews, 4, 231-234.

Mazzoni, G., Loftus, E. F. \& Kirsch, I. (2001). Changing beliefs about implausible autobiographical events: A little plausibility goes a long way. Journal of Experimental Psychology: Applied, 17(1), 51-59.

Neufeld, C. B., Brust, P. G. \& Stein, L. M. (no prelo). Adaptação de um método de investigação do impacto da emoção na memória. Revista Psico-USF.

Neufeld, C. B. \& Stein, L. M. (2003). Falsas memórias em préescolares: uma investigação experimental e suas implicações clínicas. Em M. Z. S. Brandão, F. C. S. Conte, F. S. Brandão, Y. K. Ingermann, C. B. Moura, V. M. Silva \& S. M. Oliane (Orgs.), Sobre comportamento e cognição: clínica, pesquisa e aplicação (Vol. 12, pp. 453-468). Santo André: ESETec.

Nie, N. H., Hull, C. H. \& Bent, D. H. (2003). Statistical Package for Social Sciences (Versão 12.0) [Software computacional]. São Paulo: SPSS Inc.

Nygaard, M. L. C., Feix, L. F. \& Stein, L. M. (2006). Contribuições da psicologia cognitiva para a oitiva da testemunha: avaliando a eficácia da entrevista cognitiva. Revista do Instituto Brasileiro de Ciências Criminais, 61, 147-180.

Pergher, G. K., Grassi-Oliveira, R., Ávila, L. M. \& Stein, L. M. (2005). Memória, humor e emoção. Revista de Psiquiatria do Rio Grande do Sul, 28(1), 5-12.

Pergher, G. K., Grassi-Oliveira, R. \& Stein, L. M. (2005). Memória no TEPT: dos fundamentos científicos às implicações terapêuticas. Em R. M. Caminha (Ed.), Transtorno de Estresse Pós-Traumático: da neurobiologia à terapia cognitiva (Vol. 1, pp. 105-121). São Paulo: Casa do Psicólogo.

Pergher, G. K., Stein, L. M. \& Wainer, R. (2004). Estudos sobre a memória na depressão: achados e implicações para a terapia cognitiva. Revista de Psiquiatria Clínica, 31(2), 82-90.

Quevedo, J., Sant'Anna, M. K., Madruga, M., Lovato, I., de-Paris, F., Kapczinski, F., Izquierdo, I. \& Cahill, L. (2003). Differential effects of emotional arousal in short and long-term memory in 
healthy adults. Neurobiology of Learning and Memory, 79, 132-135.

Raskin, D. \& Esplin, P. (1991). Assessment of children's statements of sexual abuse. In J. L. Doris (Ed.), The suggestibility of children's recollections (pp. 153-164). Washington: American Psychology Association.

Reyna, V. F. \& Brainerd, C. J. (1995). Fuzzy-trace theory: An interim synthesis. Learning and Individual Differences, 7, 1-75.

Safer, M. A., Levine, L. J. \& Drapalski, A. L. (2002). Distortion in memory for emotions: The contributions of personality and post-event knowledge. Personality and Social Psychology Bulletin, 28(11), 1495-1507.

Saywitz, K., Goodman, G., Nicholas, G. \& Moan, S. (1991). Children's memory of a physical examination involving genital touch: Implications for reports of child sexual abuse. Journal of Consulting and Clinical Psychology, 5, 682-691.

Stein, L. M. \& Memon, A. (2006). Testing the efficacy of the cognitive interview in a developing country. Journal of Applied Cognitive Psychology, 20, 597-605.

Stein, L. M. \& Neufeld, C. B. (2001). Falsas memórias: porque lembramos de coisas que não aconteceram? Arquivos de Ciência Saúde Unipar, 5(2), 179-186.
Stein, L. M. \& Nygaard, M. L. C. (2003). A memória em julgamento: uma análise cognitiva dos depoimentos testemunhais. Revista do Instituto Brasileiro de Ciências Criminais, 11(43), 151-164.

Stein, L. M. \& Reyna, V. F. (1997). False memories and judgments in a juror-type situation: Fuzzy-trace theory analysis. Paper presented at the JDM Meeting, U.S.A.

Titcomb, A. L. \& Reyna, V. F. (1995). Memory interference and misinformation effects. In F. N. Dempster \& C. J. Brainerd (Eds.), New perspectives on interference and inhibition in cognition. San Diego: Academic.

Warren, A. R. \& Lane, P. (1995). Effects of timing and type of questioning on eyewitness accuracy and suggestibility. In M. S. Zaragoza, J. R. Graham, G. C. Hall, R. Hirschman \& Y. S. BenPorath (Eds.), Memory and testimony in the child witness (pp. 44-60). Thousand Oaks: Sage.

Recebido em 18/02/2007

Aceito em 11/12/2007

Endereço para correspondência : Lilian Milnitsky Stein. Av. Ipiranga, 6681, Prédio 11, sala 933, CEP 90619-900, Porto AlegreRS.E-mail: lilian@pucrs.br 